\title{
Enhanced recovery after spine surgery: review of the literature
}

\author{
Marco V. Corniola, MD, ${ }^{1}$ Bertrand Debono, MD, ${ }^{2}$ Holger Joswig, MD, ${ }^{1}$ \\ Jean-Michel Lemée, MD, PhD, ${ }^{1}$ and Enrico Tessitore, MD'1 \\ 1Department of Neurosurgery, Spine Unit, Geneva University Hospitals, Geneva, Switzerland; and 'Department of Neurosurgery, \\ CAPIO-Clinique des Cèdres, Cornebarrieu, France
}

The concept of Enhanced Recovery After Surgery (ERAS) entails recovery facilitation of patients who undergo surgery through the implementation of a multidisciplinary and multimodal perioperative care approach. By its application, ERAS improves the overall functional outcome after surgery while maintaining high standards of care. A review of the essential aspects of ERAS in spine surgery was undertaken. Special consideration was given to the risks and benefits for patients and caregivers, as well as the medical and economical aspects of this concept.

https://thejns.org/doi/abs/10.3171/2019.1.FOCUS18657

KEYWORDS enhanced recovery after surgery; fast-track surgery; spine surgery

$\mathrm{T}$ HE concept of Enhanced Recovery After Surgery (ERAS), also known as "fast-track surgery," was introduced by Kehlet in $1997 . .^{16}$ It conceptualizes a multidisciplinary and multimodal perioperative care approach that aims to hasten the recovery of patients undergoing surgery. Since then, ERAS has quickly been recognized worldwide and is now promoted by both national and international dedicated surgical societies and hospitals. ${ }^{20}$

The ERAS concepts were first applied to abdominal surgery when minimally invasive laparoscopy was shown to be superior to laparotomy..$^{19}$ Following laparoscopy, patients reported less pain and their overall hospital stays were significantly shortened. At the same time, postoperative complications, such as ileus, were reduced while outcomes were similar. ${ }^{12}$ In the past decade, ERAS has been the object of more than 3000 PubMed-listed publications in various surgical fields; however, it is poorly discussed in the neurosurgical domain where it has been applied to spinal surgery over the last few years. ${ }^{32}$ Case series of outpatient microdiscectomies for lumbar disc herniation ${ }^{6}$ as well as studies of fast-track lumbar fusion procedures ${ }^{32}$ have been published. As in abdominal surgery, the use of minimally invasive spine surgery (MISS) ${ }^{33}$ preceded the introduction of ERAS in spine surgery.

The essential aspects of ERAS in spine surgery are reviewed in this paper. Special consideration was given to the risks and benefits for patients and caregivers, as well as to the medical and economical aspects.

\section{Methods}

We systematically and comprehensively reviewed the published literature on ERAS using the search function in MEDLINE (US National Library of Medicine), the Cochrane library, EMBASE, ScienceDirect, and Google Scholar, following the PRISMA guidelines on searching for articles. ${ }^{23}$ The following keywords were used: "ERAS," "Enhanced Recovery After Surgery," and "spine surgery." Only English-language articles were considered. Reviewing the reference lists of articles with relevant abstracts obtained from the search results revealed additional studies. Exclusion criteria were publications other than original reports and redundant reporting of a single data set. The

ABBREVIATIONS EBL = estimated blood loss; ERAS = Enhanced Recovery After Surgery; MISS = minimally invasive spine surgery; TLIF = transforaminal lumbar interbody fusion.

SUBMITTED November 29, 2018. ACCEPTED January 22, 2019.

INCLUDE WHEN CITING DOI: 10.3171/2019.1.FOCUS18657. 


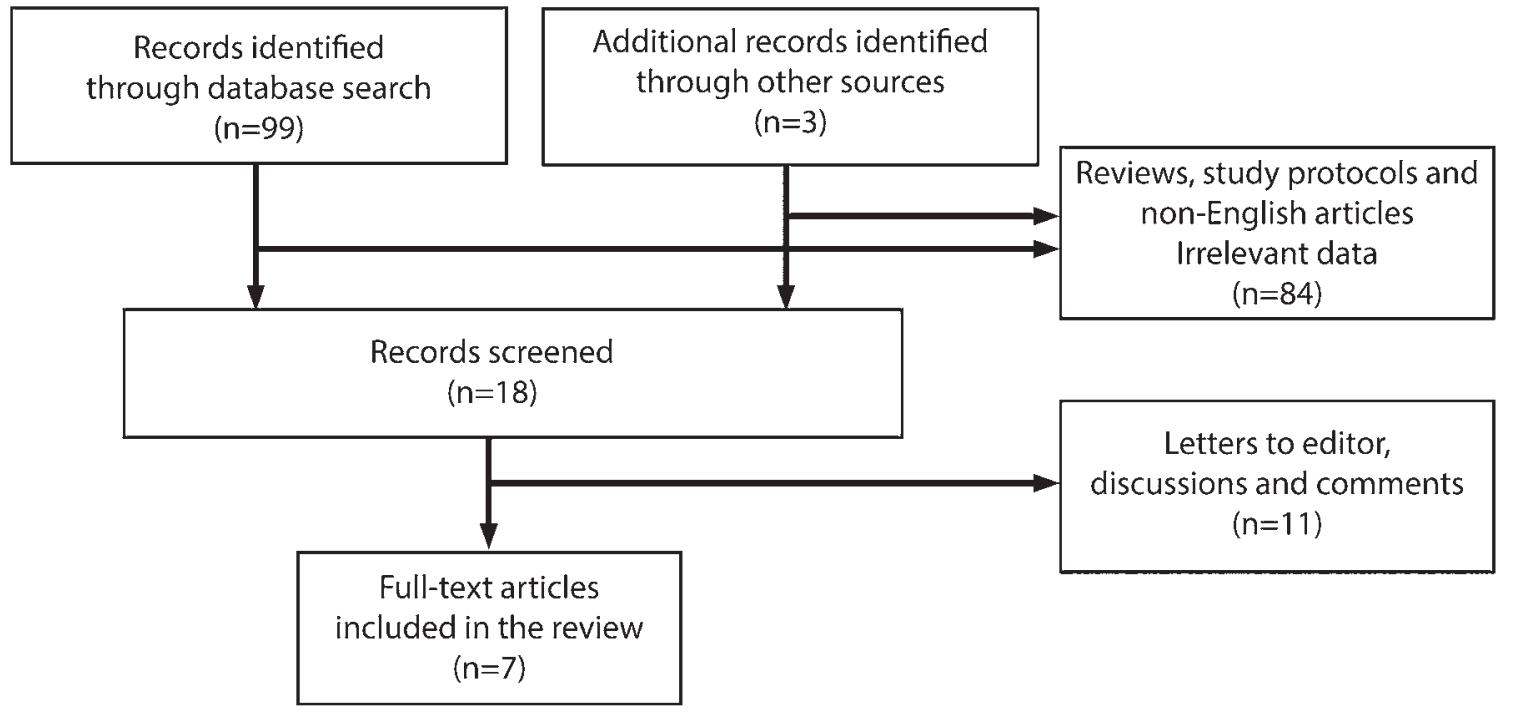

FIG. 1. Flow chart of the literature review selection process according to the PRISMA guidelines.

selection process according to the PRISMA guidelines is shown in Fig. 1. Results are summarized in Table 1 and in the following paragraphs.

\section{Results}

\section{The Concept of ERAS: A Major Shift in the Paradigm}

The implementation of ERAS in the global management of patients can be subdivided into pre-, peri-, and postoperative periods, each of which has distinct changes compared to traditional management. ${ }^{6,9,29,32}$ Here, we summarize the main concepts for each of these three phases of patient management, which are also listed in Fig. 2.

\section{Preoperative Period: Patient Education, Anxiety Management, and Fasting}

ERAS begins as early as the preoperative period ${ }^{2}$ during patient counseling with respect to the type of surgery to be performed and its potential complications in particular. Providing comprehensive information to patients about the hospital stay, care trajectory, and postdischarge nursing and treatment is crucial. Given the fact that wellinformed patients have a better postoperative outcome, realistic expectations should be set before surgery in wellselected patients ${ }^{35}$ in order to avoid later dissatisfaction.

A simplified patient preparation is preferable. . $28,32,33^{2}$ Prolonged fasting should be avoided as it has been proven to exert negative effects on the metabolism and the musculature.$^{19}$ Eating is allowed until 6 hours prior to surgery, and clear liquids are permitted even up to 2 hours before. Furthermore, carbohydrate supplementation is recommended. ${ }^{11}$

\section{Intraoperative Period: Sedation and Awake Surgery}

In six of seven publications, regular sedation was used during surgery. In the publication by Wang et al., awake surgery was used as an adjunct to ERAS. ${ }^{32,33}$ The rationale for regional instead of general anesthesia is to avoid complications linked to orotracheal intubation, ${ }^{32,33}$ providing direct patient neuromonitoring and avoiding perturbations in homeostasis. ${ }^{32}$ It implies that the surgeons have limited time to work on a patient; the airways are not secured and thus close monitoring is required. In both awake and sedation surgeries alike, controlled perioperative hydration is advised, especially in older patients. Normotonic saline solution is retained in the body, leading to an undesired positive water balance. ${ }^{19}$ Postoperative hypotension is managed with minimal fluid supplementation, whereas vasopressors are applied only if necessary. Overall, this results in a neutral fluid net effect. ${ }^{19}$

\section{Postoperative Care and Outpatient Follow-Up: Central Role of Fast-Track Nurse}

Patients are encouraged to eat and drink within hours after surgery. ${ }^{32}$ If no complications emerge, the patient is mobilized and rapidly discharged home. If required, hospitalization can be organized in a specific outpatient unit. ${ }^{6}$ Outpatient short-term follow-up is advised and can be conducted via phone interviews by a fast-track nurse who complements the regular follow-up in clinics. ${ }^{6}$

\section{Preceding ERAS: Development of MISS}

The use of MISS techniques aids in accelerating patient recovery after treatment even though such techniques are not systematically reported in the ERAS protocols. The conventional open midline approach to the thoracolumbar spine enables straightforward access to the posterior bony elements. However, detachment of the intrinsic spinal muscles results in muscular atrophy and augmented postoperative pain. This, in turn, leads to postoperative morbidity and impaired functional outcome. ${ }^{5}$ Moreover, blood loss related to the approach may require more blood transfusions, a longer hospital stay, and a greater need for revision surgery due to hematomas and infections, especially in older patients. ${ }^{5,10}$ In the articles reviewed, MISS was not exclusively used (Table 1).

Because of their potential in reducing surgical inva- 
TABLE 1. Literature review of studies on ERAS in spine surgery

\begin{tabular}{|c|c|c|c|c|c|c|c|c|c|}
\hline Authors \& Year & $\begin{array}{l}\text { No. } \\
\text { of } \\
\text { Pts }\end{array}$ & $\begin{array}{l}\text { Same-Day } \\
\text { Discharge }\end{array}$ & $\begin{array}{l}\text { Awake } \\
\text { Surgery }\end{array}$ & Surgical Procedure & MISS & $\begin{array}{l}\text { Mean Op Time } \\
\text { (min) }\end{array}$ & $\begin{array}{c}\text { Mean } \\
\text { Blood } \\
\text { Loss }(\mathrm{ml})\end{array}$ & Mean LOS & $\begin{array}{l}\text { Fusion } \\
\text { Rate }\end{array}$ \\
\hline Eckman et al., 2014 & 808 & Yes & No & 1- or 2-level TLIF & Yes & - & - & Outpatient & - \\
\hline Chin et al., 2015 & 16 & Yes & No & 1-level fusion & No & $124.85 \pm 7.10$ & $161 \pm 32$ & Outpatient & $87.5 \%$ \\
\hline Wang et al., 2017 & 42 & No & Yes & 1- or 2-level TLIF & Yes & $94.6 \pm 22.4$ & $66 \pm 30$ & $1.29 \pm 0.9$ nights & - \\
\hline Debono et al., 2017 & 201 & Yes & No & 1-level microdiscectomy & No & - & - & $10 \mathrm{hrs} 12$ mins & - \\
\hline Soffin et al., 2018 & 61 & Yes & No & Noninstrumented lumbar surgery & No & - & - & 279 mins (195-298) & - \\
\hline Grasu et al., 2018 & 41 & No & No & $\begin{array}{l}\text { Instrumented \& noninstrumented } \\
\text { spine surgery }\end{array}$ & Yes & $263.9 \pm 128.0$ & - & $6.3 \pm 2.2$ & - \\
\hline $\begin{array}{l}\text { Venkata \& van } \\
\text { Dellen, } 2018\end{array}$ & 246 & Yes & No & $\begin{array}{l}\text { Noninstrumented lumbar \& cervi- } \\
\text { cal surgery }\end{array}$ & No & - & - & Outpatient & - \\
\hline
\end{tabular}

LOS $=$ length of stay; Pts $=$ patients $;-=$ no data available.

siveness, the major concepts of MISS to be considered in ERAS are as follows:

1) Minimizing estimated blood loss (EBL). Studies report a lower average EBL in minimally invasive lumbar fusion surgery than occurs in the conventional open technique. ${ }^{3,15,27}$ For example, Archavlis et al. lowered EBL to $185 \pm 140 \mathrm{ml}$ (mean \pm standard deviation) in an MISS spondylolisthesis group versus $255 \pm 468 \mathrm{ml}$ in the open group. ${ }^{3}$ Considering that transfusions carry specific risks (e.g., alloreactions, disease transmission, limited availability), restricting blood loss is an important asset of MISS ${ }^{5,10,27}$ and should be considered in ERAS.

2) Minimizing muscular trauma. In MISS, detachment of the musculature from bone is no longer necessary. This reduces the inflammatory reaction and muscle mass loss. Fan et al. compared patients undergoing single-level posterior lumbar interbody fusion via an open and an MISS approach. ${ }^{8}$ Patients operated on using the open approach

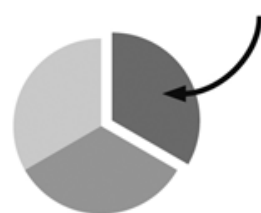

Preoperative period

- Pre-admission counseling

- Evaluation and optimization of organ dysfunction

- Smoking reduction if applicable

- Nutritional support and micronutrient supplementation

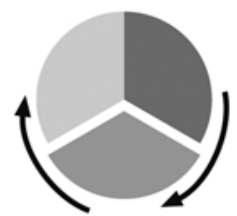

Intraoperative period

- Prophylactic antibiotics

- No pre-medication

- Regional anesthesia techniques

- Short-acting anesthesia agents, avoid opioids

- Balanced fluid/sodium
administration
- Minimally invasive surgery:
short incision,
minimal drain usage

- No NG tubes, no urinary catheter

- Hypothermia prevention, warm air body heating

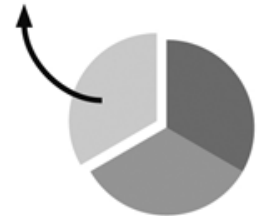

\section{Postoperative period}

- Good pain control, minimal opioids usage

- Balanced fluids with minimal i.v. therapy

- Thromboembolic and N/V prophylaxis

- Early drain, tube and catheter removal

- Early mobilization and oral feeding, stimulation of gut mobility

- Audit of complications, close FU

intravenous; NG = nasogastric; NSAIDs = nonsteroidal antiinflammatory drugs; N/V = nausea and vomiting. 
had a significantly reduced multifidus muscle cross-sectional area (CSA) on postoperative MRI, which was not the case for MISS. With both procedures, increased fatty infiltration was observed but was more pronounced in the patients operated on using the open approach. ${ }^{8}$ In a similar vein, Kim et al. reported a significant decrease in the multifidus muscle CSA after open pedicle screw fixation, which was not seen in the percutaneous pedicle screw fixation group. ${ }^{18}$ Shunwu et al. demonstrated that patients undergoing minimally invasive transforaminal lumbar interbody fusion (TLIF) had lower serum creatinine kinase levels than those undergoing open TLIF. ${ }^{26}$ Altogether, these factors support the adjunct use of minimally invasive percutaneous pedicular fixation in ERAS.

3) Faster postoperative mobilization. According to Seng et al., after minimally invasive TLIF, patients had a faster time in resuming ambulation by a factor of two (1.5 vs 3 days) compared to patients in the open TLIF group. ${ }^{25}$ Shunwu et al. had similar results. ${ }^{26}$

4) There are also fewer postoperative infections following MISS. By avoiding the creation of dead space in between the skin and the muscular plane using small incisions, surgical site infections (SSIs) can be significantly reduced, as shown in a multicenter study by McGirt et al. ${ }^{22}$ Fewer SSIs were observed in an MISS group of patients undergoing percutaneous 2-level TLIF, which turned out to be a cost savings as well.

5) Postoperative pain management. MISS has been shown to significantly reduce opioid consumption in the postoperative period. Adogwa et al. demonstrated how the duration of postoperative opioid use can be significantly shortened in patients undergoing minimally invasive TLIF ( 2 weeks) versus open TLIF (4 weeks). ${ }^{1}$ In another study, the total mean morphine use for minimally invasive TLIF (17.4 mg) was less than that for open TLIF (35.7 $\mathrm{mg}) .^{24}$ Similar observations were made by Isaacs et al. ${ }^{14}$ and Seng et al. ${ }^{25}$

6) Cost reduction. Going hand in hand with faster mobilization, shorter hospitalizations were noted in patients after minimally invasive TLIF than in patients who underwent open fusion (3.6 vs 5.9 days, $\mathrm{p}<0.001$; refer to point 3 above) ${ }^{25}$ Singh et al. conducted a financial analysis of minimally invasive TLIF procedures and showed that hospital costs can be lowered by using MISS techniques (\$19,512 in MISS vs $\$ 23,550$ in open TLIF). ${ }^{27}$ In their study, implant costs were similar in both groups and accounted for two-thirds of the direct hospital costs in the MISS cohort and half of the costs in the open group. Hospital reimbursements were higher for the standard open TLIF patients than the MISS group. ${ }^{27}$

\section{Discussion}

In theory, the purpose of ERAS is to increase patient comfort and satisfaction by reducing the invasiveness of the surgical procedure ${ }^{31}$ and its associated complications, as well as by shortening the overall length of stay. In reality, the application of ERAS in spine surgery faces many problems involving caregiver training, the development of less invasive surgical techniques, and the conduct of accurate follow-up. Moreover, difficulties with hospital re- imbursements need to be considered. From a patient perspective, high compliance is warranted as ERAS demands strict adherence to the treatment plan. The implementation of ERAS in daily clinical practice is challenged by patients' and surgeons' fears of complications and beliefs, as well as cultural and environmental factors. Moreover, a comprehensive and multimodal approach to postoperative pain, nausea, and vomiting should be fully integrated. ${ }^{21}$ Great efforts will have to be made in the next decades to ensure good, accurate clinical practice as well as to generate more prospective data.

\section{MISS, Awake Surgery, and the Outpatient Setting in ERAS}

The MISS technique-although proven to be efficacious in terms of reducing postoperative pain-is not systemically applied for ERAS. As can be seen from Table 1 , four groups did not incorporate the technique. This is paradoxical since MISS and ERAS both aim to reduce surgical invasiveness. Without MISS, ERAS loses its relevance. Thus, MISS has to be viewed as the specific surgical arm of a more global ERAS concept applied to spine surgery. Similarly, only one author group performed ERAS-aligned awake instrumented lumbar procedures, ${ }^{32}$ which requires well-trained surgeons as well as anesthesiologists.

The ERAS concept is easily applicable to noninstrumented spinal procedures such as lumbar disc herniation or spinal stenosis but seems more difficult to put into practice when it comes to complex spine cases. ERAS does not require the patient to be discharged directly after surgery. As patient safety comes first, the aim for same-day discharge should not be the priority in ERAS guidelines.

Finally, caregivers must not envision ERAS as an accelerated discharge protocol and should certainly not confuse it with a simple "fast-track" surgery. ${ }^{17}$

\section{ERAS and Instrumented Spinal Procedures}

Chin et al. ${ }^{4}$ first described the implementation of ERAS in instrumented spinal procedures. In their case series, 16 patients underwent outpatient open, single-level TLIF. The operations lasted $125 \pm 7$ min on average with an EBL of $161 \pm 32 \mathrm{ml}$. The mean low-back visual analog scale score of $8.4 \pm 0.37$ was reduced significantly to $4.96 \pm$ 0.73 . Functional outcome, as measured with the Oswestry Disability Index, was significantly improved from $52.71 \pm$ 0.04 preoperatively to $37.43 \pm 0.06$ postoperatively. The fusion rate was $87.5 \% .^{4}$

Eckman et al. retrospectively compared 808 outpatient minimally invasive TLIF procedures to inpatient procedures.7 These authors reported similar outcomes between the two groups except for a lower visual analog scale score for leg pain in the inpatients. In this study, however, patients 65 years or older were selected for inhospital stays.

Wang et al. published the first study on awake, endoscopic TLIF in 10 patients. ${ }^{33}$ The average operating time was $113.5 \pm 6.3 \mathrm{~min}$ with a mean blood loss of $65 \pm 38 \mathrm{ml}$. Contrary to the report by Debono et al., the procedures were not outpatient. Functional status and pain were both improved. A second report on 42 consecutive cases was recently published by the same group ${ }^{32}$ and represents the 
largest study on awake minimally invasive TLIF that implemented an ERAS approach in the daily clinical practice. Following the same methodology and with results comparable to those in their first publication, the authors showed that ERAS in spinal fusion was effective and feasible with favorable early functional outcomes; significant improvement in the Oswestry Disability Index from $40 \pm$ 13 to $17 \pm 11(\mathrm{p}<0.01)$ was noted. ${ }^{32}$

\section{ERAS in Oncological Patients}

The ERAS concept is even more valid for cancer patients. Debilitated cancer patients who are candidates for surgery may benefit from the ERAS approach by having a quicker surgical recovery and more rapid movement to rehabilitation or complementary nonsurgical treatments. Furthermore, MISS techniques are now strongly recommended in cancer patients presenting with spinal involvement. Recently, Grasu et al. reported on the use of the ERAS concept in oncological patients undergoing spinal procedures. ${ }^{11}$ By comparing the standard protocol with the ERAS approach in 56 and 41 patients, respectively, the authors demonstrated the feasibility and effectiveness of ERAS on pain control in oncological spine surgery patients. Those patients who had been treated with the ERAS approach had better pain scores and less opioid consumption than the patients who had undergone surgery before the implementation of ERAS. Although they were retrospective, these studies showed that ERAS can be applied to cancer patients, in whom reduced surgical invasiveness and early recovery are key to rapidly proceed to complementary treatment and rehabilitation.

\section{Medical and Healthcare Costs}

As healthcare systems differ from one country to another, the analysis of medical costs in ERAS is difficult and entails bias, limiting the discussion on medico-economics. Moreover, current practice ensures hospital reimbursement for inpatient management and does not provide proper financial compensation when it comes to ERAS. However, some preliminary data ${ }^{6,34}$ have shown that despite major flaws, ERAS can save financial resources. Medical infrastructures are not always designed for the implementation of ERAS. Thus, the potential for savings is probably underestimated. In addition, ERAS without MISS introduces bias; that is, patients undergoing MISS or open surgery cannot be directly compared, as previously noted by Wang et al. ${ }^{34}$ Their patients undergoing MISS were more likely to be young and healthy.

Medical costs were investigated in noninstrumented as well as instrumented MISS and ERAS procedures. Debono et al. showed how costs can be halved by introducing outpatient microdiscectomy ${ }^{6}(€ 224.08$ vs $€ 520.38$ for inpatient procedures). Wang et al. showed that the mean costs for a single-level fusion MISS case $(\$ 29,187 \pm \$ 461)$ are marginally cheaper than for open surgery $(\$ 29,947 \pm$ $\$ 324 ; p=0.55)$. For 2-level surgeries, the mean costs of MISS were $\$ 33,879 \pm \$ 521$ as compared to $\$ 35,984 \pm \$ 269$ for open surgery $(\mathrm{p}=0.002) .{ }^{34}$ As previously mentioned, it should be noted that the implementation of MISS has an impact on hospital reimbursement. In a cost analysis of open versus minimally invasive TLIF, Singh et al. showed that hospital reimbursement was $\$ 6248$ higher for open TLIF cases than for MISS cases. This resulted in a \$2210 greater hospital profit in favor of the open TLIF group. ${ }^{27}$ Thus, the implementation of ERAS is probably impeded by current reimbursement policies.

\section{ERAS in the Future}

Since lumbar degenerative pathologies account for a large healthcare burden in Western countries, ${ }^{13}$ the optimization of treatment costs via the ERAS system is warranted. Besides preventing physiological stress related to surgery and allowing a quicker return to normal daily activities, ERAS provides tools to improve functional outcomes. ${ }^{31}$ Developments in new surgical and anesthesiological techniques that will eventually propagate a rapid worldwide spread of the ERAS concept in spine surgery must be undertaken. Moreover, not only surgical disciplines are concerned in the improvement of ERAS in spine surgery. From a strictly economical point of view, surgeons should provide robust economical and clinical data to challenge the current reimbursement policies. The reduced rate of complications, the adherence and active participation of patients, and the decreased length of stay may lead to lower healthcare costs. In addition, as patients are discharged more quickly, patient turnover is increased. All of these considerations render the implementation of ERAS within the field of neurosurgery interesting for governments, administrators, caregivers, and patients, especially since ERAS has already been proven efficient in other surgical specialties.

\section{Study Strengths and Limitations}

There is a dearth of information in the literature about ERAS in spine surgery. This is especially true for ERAS in neurosurgery. Surely, this weakens any review of the literature on the topic, but it also reveals the need for a thorough exploration of ERAS in spine surgery.

The economical factor will probably be the main point to work on in the future in order to eventually expand ERAS in spine surgery. The most important contributions to the economical analysis of MISS and ERAS are provided by Wang et al..$^{34}$ and Debono et al. ${ }^{6}$ The publication by Wang et al. takes into consideration minimally invasive surgery in lumbar spine fusion, which is only one technical aspect of the ERAS approach. The publication by Debono et al. on outpatient microdiscectomy is more in the spirit of ERAS. However, it does not provide data on more complex cases, which is a slight limitation for cost analysis. These two publications are of great value and serve as a good starting point to expand the economical concept further; however, they introduce a selection bias, as older patients and/or less healthy patients have been excluded from the MISS and/or ERAS programs. Also, international comparison is difficult because of the very different healthcare and reimbursement systems from one country to another.

The strategies to develop to engage hospital administrators in ERAS should also be discussed further. This illustrates the challenge of such a paradigm shift toward 
ERAS in an international milieu. In France, Switzerland, and probably all of Northern Europe, healthcare systems are more in favor of outpatient management-for economical reasons certainly, but also due to cultural beliefs. And this can also vary between public and private hospitals.

\section{Conclusions}

The ERAS approach is a safe and effective tool to reduce the overall invasiveness of spine surgery, as well as its related costs through shortened hospitalizations, while maintaining a high standard of care and patient comfort. However, medico-economical considerations need to be tailored to the healthcare environment. The education of patients and caregivers is paramount. To date, the results reported in the literature are promising, but further studies are needed to justify an expansion of ERAS in the field of spine surgery.

\section{Acknowledgments}

The authors thank Nadia Fries for reviewing the manuscript.

\section{References}

1. Adogwa O, Parker SL, Bydon A, Cheng J, McGirt MJ: Comparative effectiveness of minimally invasive versus open transforaminal lumbar interbody fusion: 2-year assessment of narcotic use, return to work, disability, and quality of life. J Spinal Disord Tech 24:479-484, 2011

2. Ali ZS, Ma TS, Ozturk AK, Malhotra NR, Schuster JM, Marcotte PJ, et al: Pre-optimization of spinal surgery patients: Development of a neurosurgical enhanced recovery after surgery (ERAS) protocol. Clin Neurol Neurosurg 164:142-153, 2018

3. Archavlis E, Carvi y Nievas M: Comparison of minimally invasive fusion and instrumentation versus open surgery for severe stenotic spondylolisthesis with high-grade facet joint osteoarthritis. Eur Spine J 22:1731-1740, 2013

4. Chin KR, Coombs AV, Seale JA: Feasibility and patientreported outcomes after outpatient single-level instrumented posterior lumbar interbody fusion in a surgery center: preliminary results in 16 patients. Spine (Phila Pa 1976) 40:E36-E42, 2015

5. Corniola MV, Stienen MN, Tessitore E, Schaller K, Gautschi OP: [Minimally invasive spine surgery: past and present.] Rev Med Suisse 11:2186-2189, 2015 (Fr)

6. Debono B, Sabatier P, Garnault V, Hamel O, Bousquet P, Lescure JP, et al: Outpatient lumbar microdiscectomy in france: from an economic imperative to a clinical standard-an observational study of 201 cases. World Neurosurg 106:891-897, 2017

7. Eckman WW, Hester L, McMillen M: Same-day discharge after minimally invasive transforaminal lumbar interbody fusion: a series of 808 cases. Clin Orthop Relat Res 472:1806-1812, 2014

8. Fan SW, Hu ZJ, Fang XQ, Zhao FD, Huang Y, Yu HJ: Comparison of paraspinal muscle injury in one-level lumbar posterior inter-body fusion: modified minimally invasive and traditional open approaches. Orthop Surg 2:194-200, 2010

9. Fearon KC, Ljungqvist O, Von Meyenfeldt M, Revhaug A, Dejong $\mathrm{CH}$, Lassen $\mathrm{K}$, et al: Enhanced recovery after surgery: a consensus review of clinical care for patients undergoing colonic resection. Clin Nutr 24:466-477, 2005

10. Goldstein CL, Macwan K, Sundararajan K, Rampersaud YR: Comparative outcomes of minimally invasive surgery for posterior lumbar fusion: a systematic review. Clin Orthop Relat Res 472:1727-1737, 2014
11. Grasu RM, Cata JP, Dang AQ, Tatsui CE, Rhines LD, Hagan $\mathrm{KB}$, et al: Implementation of an Enhanced Recovery After Spine Surgery program at a large cancer center: a preliminary analysis. J Neurosurg Spine 29:588-598, 2018

12. Greco M, Capretti G, Beretta L, Gemma M, Pecorelli N, Braga M: Enhanced recovery program in colorectal surgery: a meta-analysis of randomized controlled trials. World J Surg 38:1531-1541, 2014

13. Hofstetter CP, Hofer AS, Wang MY: Economic impact of minimally invasive lumbar surgery. World J Orthop 6:190201, 2015

14. Isaacs RE, Podichetty VK, Santiago P, Sandhu FA, Spears J, Kelly K, et al: Minimally invasive microendoscopy-assisted transforaminal lumbar interbody fusion with instrumentation. J Neurosurg Spine 3:98-105, 2005

15. Joswig H, Richter H, Haile SR, Hildebrandt G, Fournier JY: Introducing interlaminar full-endoscopic lumbar diskectomy: a critical analysis of complications, recurrence rates, and outcome in view of two spinal surgeons' learning curves. J Neurol Surg A Cent Eur Neurosurg 77:406-415, 2016

16. Kehlet H: Multimodal approach to control postoperative pathophysiology and rehabilitation. Br J Anaesth 78:606617, 1997

17. Kehlet H, Thienpont E: Fast-track knee arthroplasty-status and future challenges. Knee 20 (Suppl 1):S29-S33, 2013

18. Kim DY, Lee SH, Chung SK, Lee HY: Comparison of multifidus muscle atrophy and trunk extension muscle strength: percutaneous versus open pedicle screw fixation. Spine (Phila Pa 1976) 30:123-129, 2005

19. Ljungqvist O: ERAS-Enhanced Recovery After Surgery: moving evidence-based perioperative care to practice. JPEN J Parenter Enteral Nutr 38:559-566, 2014

20. Ljungqvist O, Scott M, Fearon KC: Enhanced Recovery After Surgery: a review. JAMA Surg 152:292-298, 2017

21. Mathiesen O, Dahl B, Thomsen BA, Kitter B, Sonne N, Dahl JB, et al: A comprehensive multimodal pain treatment reduces opioid consumption after multilevel spine surgery. Eur Spine J 22:2089-2096, 2013

22. McGirt MJ, Parker SL, Lerner J, Engelhart L, Knight T, Wang MY: Comparative analysis of perioperative surgical site infection after minimally invasive versus open posterior/ transforaminal lumbar interbody fusion: analysis of hospital billing and discharge data from 5170 patients. J Neurosurg Spine 14:771-778, 2011

23. Moher D, Liberati A, Tetzlaff J, Altman DG: Preferred reporting items for systematic reviews and meta-analyses: the PRISMA statement. J Clin Epidemiol 62:1006-1012, 2009

24. Peng CW, Yue WM, Poh SY, Yeo W, Tan SB: Clinical and radiological outcomes of minimally invasive versus open transforaminal lumbar interbody fusion. Spine (Phila Pa 1976) 34:1385-1389, 2009

25. Seng C, Siddiqui MA, Wong KP, Zhang K, Yeo W, Tan SB, et al: Five-year outcomes of minimally invasive versus open transforaminal lumbar interbody fusion: a matched-pair comparison study. Spine (Phila Pa 1976) 38:2049-2055, 2013

26. Shunwu F, Xing Z, Fengdong Z, Xiangqian F: Minimally invasive transforaminal lumbar interbody fusion for the treatment of degenerative lumbar diseases. Spine (Phila Pa 1976) 35:1615-1620, 2010

27. Singh K, Nandyala SV, Marquez-Lara A, Fineberg SJ, Oglesby M, Pelton MA, et al: A perioperative cost analysis comparing single-level minimally invasive and open transforaminal lumbar interbody fusion. Spine J 14:1694-1701, 2014

28. Soffin EM, Vaishnav AS, Wetmore D, Barber L, Hill P, Gang $\mathrm{CH}$, et al: Design and implementation of an Enhanced Recovery After Surgery (ERAS) Program for minimally invasive lumbar decompression spine surgery: initial experience. Spine (Phila Pa 1976) [epub ahead of print], 2018 
29. Taurchini M, Del Naja C, Tancredi A: Enhanced recovery after surgery: a patient centered process. J Vis Surg 4:40, 2018

30. Venkata HK, van Dellen JR: A perspective on the use of an enhanced recovery program in open, non-instrumented day surgery for degenerative lumbar and cervical spinal conditions. J Neurosurg Sci 62:245-254, 2018

31. Wainwright TW, Immins T, Middleton RG: Enhanced recovery after surgery (ERAS) and its applicability for major spine surgery. Best Pract Res Clin Anaesthesiol 30:91-102, 2016

32. Wang MY, Chang PY, Grossman J: Development of an Enhanced Recovery After Surgery (ERAS) approach for lumbar spinal fusion. J Neurosurg Spine 26:411-418, 2017

33. Wang MY, Grossman J: Endoscopic minimally invasive transforaminal interbody fusion without general anesthesia: initial clinical experience with 1-year follow-up. Neurosurg Focus 40(2):E13, 2016

34. Wang MY, Lerner J, Lesko J, McGirt MJ: Acute hospital costs after minimally invasive versus open lumbar interbody fusion: data from a US national database with 6106 patients. J Spinal Disord Tech 25:324-328, 2012

35. Yee A, Adjei N, Do J, Ford M, Finkelstein J: Do patient expectations of spinal surgery relate to functional outcome? Clin Orthop Relat Res 466:1154-1161, 2008

\section{Disclosures}

Dr. Tessitore has received training fees from Spineart, DePuy Synthes, NuVasive, Brainlab, and Medtronic.

\section{Author Contributions}

Conception and design: Corniola, Tessitore. Acquisition of data: Corniola. Analysis and interpretation of data: Corniola. Drafting the article: all authors. Critically revising the article: all authors. Reviewed submitted version of manuscript: Corniola, Tessitore. Approved the final version of the manuscript on behalf of all authors: Corniola. Administrative/technical/material support: Tessitore. Study supervision: Corniola, Tessitore.

\section{Correspondence}

Marco V. Corniola: Geneva University Hospitals, Geneva, Switzerland.marco.corniola@hcuge.ch. 